\title{
Is It Important to Model the Impact of Blood Flow on the Dose of Drugs Delivered Transcutaneously?
}

\author{
Geraldine Clough $^{\mathrm{a}}$ Rodney Gush ${ }^{\mathrm{b}}$ \\ a School of Medicine, University of Southampton, Southampton, and ${ }^{\mathrm{b}}$ Moor Instruments, Axminster, UK
}

The assessment of endothelial responsiveness to vasodilator stimuli has long been considered a surrogate by which to evaluate cardiovascular risk. Our understanding of the endothelial phenotype in health and disease and the impact or therapeutic intervention on endothelial function are largely based on the measurement of changes in vessel diameter and/or perfusion in the peripheral vasculature following perturbation using physiological and pharmacological stimuli. These tests need to be non-invasive, reproducible, repeatable and standardized between laboratories if they are to be of clinical value [1]. They also should not modulate endothelial vascular function by themselves. At the level of the microvasculature, these tests generally involve perturbation of the vasculature using reactive hyperaemia, local warming, or introduction of pharmacological agents and the subsequent monitoring of the vasoresponse [2].

Iontophoretic delivery of vaso-active substances to the skin combined with laser Doppler flowmetry or perfusion imaging has been widely used for the assessment of vascular endothelial function in both research and clinical settings. The recognized advantages of the technique are that drug delivery is non-invasive and local, thus avoiding tissue perturbation and/or systemic drug effects, and that, by controlling the duration and magnitude of the iontophoretic current based on Coulomb's law, the dose of drug delivered to the skin is controlled.

Iontophoresis uses a small electric current to drive charged substances into the skin. Electromigration of ions during iontophoresis has also been shown to induce osmotic water flow and the convective movement of neutral or even charged molecules - a process termed electro-osmosis. It is generally held that the quantity of drug delivered depends on the magnitude and duration of the current applied, the driving force (voltage) required to achieve this dose depending on the nature of the structure of the skin and its compartments. Human skin consists of three main layers - the epidermis, dermis and hypodermis. While the stratum corneum of the epidermis, with its structured lipophilic and hydrophilic domains, constitutes the major rate-limiting layer for transdermal delivery of drugs, the dermis and its vasculature, which act as the systemic absorption site for iontophoresed drugs, present the greatest challenge when modelling transdermal iontophoretic transport.

The movement of agents into and within the dermis is subject to many variables, including diffusive and electrorepulsive forces at the epidermal barrier, protein/receptor binding and drug metabolism which together modulate the presence of 'ambient' ions in the skin that contribute to the current flow which defines drug dose.

\section{KARGER}

Fax +4161306 1234 E-Mail karger@karger.ch www.karger.com
(C) 2008 S. Karger AG, Basel

1018-1172/09/0464-0267\$26.00/0

Accessible online at:

www.karger.com/jvr
Dr. Geraldine Clough

University of Southampton School of Medicine

Southampton General Hospital

Mailpoint 887, Southampton SO16 6YD (UK)

Tel. +44 238071 4292, Fax +44 238079 4760, E-Mail gfc1@soton.ac.uk 
However, there is increasing awareness that the main challenge to modelling transdermal iontophoretic drug delivery is an understanding of the diffusive and mass transport processes within the dermis that determine the local tissue concentration and removal of substances by the blood and lymph.

Many attempts have been made at defining the rate of iontophoretic delivery to the tissue space and to develop models for a quantitative description of the in vivo transdermal transport of an iontophoresed substance $[3,4]$. The report by Tesselaar et al. [5] in this issue of the journal adds to these models and also seeks to explain why responses to total drug dose depend on the rate of drug delivery. In their study, these authors have administered acetylcholine and sodium nitroprusside to the skin of the volar forearm of healthy volunteers using a single-pulse, constant-current iontophoresis protocol. By seeking to fit both sigmoidal dose-response curves and a new time-response model to the blood flow responses, they explore the dynamics of the response to different vaso-active mediators in terms of $\mathrm{ED}_{50}$, Hill slope parameters [6] and maximum response. The model developed represents the microvascular bed as a single compartment with a zeroorder influx of the drug from the electrode and, because blood flow is a non-linear function of drug delivery, a first-order clearance of the drug from the compartment by diffusion and blood flow. Using this model, they explore the impact of clearance of drugs from the skin by the vasculature and the relationship between local drug concentration and blood flow.

The finding by Tesselaar et al. [5] that there is a significant clearance of drug out of the skin during iontophoresis is not surprising. In pharmacokinetic studies, where microdialysis sampling using small permeable probes has been used to continuously sample the tissue space to assess the transcutaneous delivery of xenobiotics, there has long been awareness that the disposition of analytes is subject to mass transport processes between the tissues and the blood [7-10]. These studies have shown that clearance of a small solute from the extravascular tissue space by the blood may increase more than 4 -fold during maximal vasodilatation in the skin [11]. Interestingly, microdialysis techniques have also been used to explore the efficacy of percutaneous delivery and to assess the pharmacokinetics of iontophoretically delivered drugs in the dermis $[12,13]$.

In the conclusion to their paper, Tesselaar et al. [5] suggest that short protocols using higher current strengths rather than low current strengths over longer periods, where clearance by the vasculature may impact more on effective dose, may be preferable. This, however, takes us into the other realm of non-specific charge effects [1416]. In some studies, delivery of acetylcholine by iontophoresis has been shown to result in an axon reflex. This axon reflex, while dependent on the experimental conditions - largely the charge density -, will contribute to the increase in cutaneous blood flow and hence distribution of active drug. The response is more pronounced in the cathodal mode that is used for sodium nitroprusside iontophoresis [17, 18]. The use of a low anodal current or of larger iontophoresis electrodes creates a smaller charge density and, therefore, the involvement of the axon reflex can be decreased. This appears contrary to the recommendations of Tesselaar et al. [5].

\section{Why Might This Be Important?}

There is considerable interest in the skin as a port of entry into the body for the systemic delivery of therapeutic agents with diverse physicochemical properties, including drugs of differing lipophilicity and molecular weight such as proteins, peptides and antisense oligonucleotides. The combination of iontophoresis with electroporation, mechanical perturbation of the epidermal barrier, chemical enhancers and ion exchange material may also offer an easier and more accurate process by which to deliver macromolecules and poorly water-soluble compounds [19]. The models of iontophoresis provide methods for assessing the local clearance of a drug, which is important if its distribution is either to be limited, for drugs for local effect, or conversely to be enhanced, for drugs to be effective systemically. The role for such models in drug monitoring will be to avoid the factors which may confound assessment of tissue concentrations.

The role for modelling in tests of microvascular mechanisms, including endothelial function, is also to avoid factors which may confound appropriate assessment of the magnitude of a given response.

\section{Conclusions}

There are many unknowns in the determination of effective dose by iontophoresis: we do not know the proportion of current conveyed by drug ions and ambient ions entering the skin, or conversely, by charged ambient ions leaving the skin. We also do not know enough about the composition of the vasculature responsible for the chang- 
es induced by the iontophoresed drug in blood flow because with laser Doppler flowmetry, where the flux signal is proportional to the product of the average speed of moving blood cells and their concentration (blood volume), fast flow of blood with a low erythrocyte concentration is equivalent to slow flow with a higher erythrocyte concentration.
The models are still useful because they move the analysis towards physiological rather than simple numerical parameters. Some models are more practical than others for clinical implementation. However, the number of unknowns warns us against overinterpretation.

\section{References}

1 Deanfield JE, Halcox JP, Rabelink TJ: Endothelial function and dysfunction: testing and clinical relevance. Circulation 2007;115: 1285-1295.

$\checkmark 2$ Cracowski JL, Minson CT, Salvat-Melis M, Halliwill JR: Methodological issues in the assessment of skin microvascular endothelial function in humans. Trends Pharmacol Sci 2006;27:503-508.

$\checkmark 3$ Nugroho AK, Della Pasqua O, Danhof M, Bouwstra JA: Compartmental modeling of transdermal iontophoretic transport. II. In vivo model derivation and application. Pharm Res 2005;22:335-346.

4 de Mul FF, Blaauw J, Aarnoudse JG, Smit AJ, Rakhorst G: Diffusion model for iontophoresis measured by laser-Doppler perfusion flowmetry, applied to normal and preeclamptic pregnancies. J Biomed Opt 2007; 12:014032.

5 Tesselaar E, Henricson J, Jonsson S, Sjöberg F: A time-response model for analysing of drug transport and blood flow response during iontophoresis of acetylcholine and sodium nitroprusside. J Vasc Res 2009;46:270277.

6 Henricson J, Tesselaar E, Persson K, Nilsson G, Sjoberg F: Assessment of microvascular function by study of the dose-response effects of iontophoretically applied drugs (acetylcholine and sodium nitroprusside) methods and comparison with in vitro studies. Microvasc Res 2007;73:143-149.
Bungay PM, Morrison PF, Dedrick RL: Steady-state theory for quantitative microdialysis of solutes and water in vivo and in vitro. Life Sci 1990;46:105-119.

-8 Singh P, Roberts MS: Effects of vasoconstriction on dermal pharmacokinetics and local tissue distribution of compounds. J Pharmaceutical Sci 1994;83:783-791.

$\checkmark 9$ Sun H, Bungay PM, Elmquist WF: Effect of capillary efflux transport inhibition on the determination of probe recovery during in vivo microdialysis in the brain. J Pharmacol Exp Ther 2001;297:991-1000.

10 Clough GF, Boutsiouki P, Church MK, Michel CC: Effects of blood flow on the in vivo recovery of a small diffusible molecule by microdialysis in human skin. J Pharmacol Exp Ther 2002;302:681-686.

11 Boutsiouki P, Thompson JP, Clough GF: Effects of local blood flow on the percutaneous absorption of the organophosphorus compound malathion: a microdialysis study in man. Arch Toxicol 2001;75:321-328.

12 Stagni G, O’Donnell D, Liu YJ, Kellogg DL, Morgan T, Shepherd AM: Intradermal microdialysis: kinetics of iontophoretically delivered propranolol in forearm dermis. J Control Release 2000;63:331-339.
3 Stagni G, O’Donnell D, Liu YJ, Kellogg DL Jr, Shepherd AM: Iontophoretic current and intradermal microdialysis recovery in humans. J Pharmacol Toxicol Methods 1999; 41:49-54.

14 Grossmann M, Jamieson MJ, Kellogg DL Jr, Kosiba WA, Pergola PE, Crandall CG, et al: The effect of iontophoresis on the cutaneous vasculature: evidence for current-induced hyperemia. Microvasc Res 1995;50:444452.

15 Ramsay JE, Ferrell WR, Greer IA, Sattar N: Factors critical to iontophoretic assessment of vascular reactivity: implications for clinical studies of endothelial dysfunction. J Cardiovasc Pharmacol 2002;39:9-17.

16 Horiuchi Y, Droog EJ, Henricson J, Wikström T, Lennquist S, Sjöberg F: Role of histamine release in nonspecific vasodilatation during anodal and cathodal iontophoresis. Microvasc Res 2004;67:192-196.

17 Ferrell WR, Ramsay JE, Brooks N, Lockhart JC, Dickson S, McNeece GM, et al: Elimination of electrically induced iontophoretic artefacts: implications for non-invasive assessment of peripheral microvascular function. J Vasc Res 2002;39:447-455.

18 Droog EJ, Henricson J, Nilsson GE, Sjoberg F: A protocol for iontophoresis of acetylcholine and sodium nitroprusside that minimises nonspecific vasodilatory effects. Microvasc Res 2004;67:197-202.

$>19$ Brown MB, Traynor MJ, Martin GP, Akomeah FK: Transdermal drug delivery systems: skin perturbation devices. Methods Mol Biol 2008;437:119-139. 